\title{
On the harmonic number expansion by Ramanujan
}

\author{
Cristinel Mortici ${ }^{*}$ and Chao-Ping Chen ${ }^{2}$
}

"Correspondence:

cmortici@valahia.ro

'Department of Mathematics,

Valahia University of Târgovişte, Bd.

Unirii 18, Târgovişte, 130082,

Romania

Full list of author information is

available at the end of the article

\section{Abstract}

Let $\gamma=0.577215664 \ldots$ denote the Euler-Mascheroni constant, and let the sequences

$$
\begin{aligned}
u_{n}= & \sum_{k=1}^{n} \frac{1}{k}-\frac{1}{2} \ln \left(n^{2}+n+\frac{1}{3}\right)-\frac{1}{r\left(n^{2}+n+\frac{1}{3}\right)+s\left(n^{2}+n+\frac{1}{3}\right)^{2}+t} \text { and } \\
v_{n}= & \sum_{k=1}^{n} \frac{1}{k}-\frac{1}{2} \ln \left(n^{2}+n+\frac{1}{3}\right) \\
& -\left(\frac{a}{\left(n^{2}+n+\frac{1}{3}\right)^{2}}+\frac{b}{\left(n^{2}+n+\frac{1}{3}\right)^{3}}+\frac{c}{\left(n^{2}+n+\frac{1}{3}\right)^{4}}+\frac{d}{\left(n^{2}+n+\frac{1}{3}\right)^{5}}\right) .
\end{aligned}
$$

The main aim of this paper is to find the values $r, s, t, a, b, c$ and $d$ which provide the fastest sequences $\left(u_{n}\right)_{n \geq 1}$ and $\left(v_{n}\right)_{n \geq 1}$ approximating the Euler-Mascheroni constant. Also, we give the upper and lower bounds for $\sum_{k=1}^{n} \frac{1}{k}-\frac{1}{2} \ln \left(n^{2}+n+\frac{1}{3}\right)-\gamma$ in terms of $n^{2}+n+\frac{1}{3}$

MSC: $11 Y 60 ; 40 \mathrm{A05} ; 33 \mathrm{~B} 15$

Keywords: Euler-Mascheroni constant; harmonic numbers; inequality; psi function; polygamma functions; asymptotic expansion

\section{Introduction}

The Euler-Mascheroni constant $\gamma=0.577215664 \ldots$ is defined as the limit of the sequence

$$
D_{n}=H_{n}-\ln n
$$

where $H_{n}$ denotes the $n$th harmonic number defined for $n \in \mathbb{N}:=\{1,2,3, \ldots\}$ by

$$
H_{n}=\sum_{k=1}^{n} \frac{1}{k}
$$

Several bounds for $D_{n}-\gamma$ have been given in the literature [1-7]. For example, the following bounds for $D_{n}-\gamma$ were established in $[3,7]$ :

$$
\frac{1}{2(n+1)}<D_{n}-\gamma<\frac{1}{2 n} \quad(n \in \mathbb{N}) .
$$

(c) 2013 Mortici and Chen; licensee Springer. This is an Open Access article distributed under the terms of the Creative Commons Attribution License (http://creativecommons.org/licenses/by/2.0), which permits unrestricted use, distribution, and reproduction in any medium, provided the original work is properly cited. 
The convergence of the sequence $D_{n}$ to $\gamma$ is very slow. Some quicker approximations to the Euler-Mascheroni constant were established in [8-21]. For example, Cesàro [8] proved that for every positive integer $n \geq 1$, there exists a number $c_{n} \in(0,1)$ such that the following approximation is valid:

$$
\sum_{k=1}^{n} \frac{1}{k}-\frac{1}{2} \ln \left(n^{2}+n\right)-\gamma=\frac{c_{n}}{6 n(n+1)}
$$

Entry 9 of Chapter 38 of Berndt's edition of Ramanujan's Notebooks [22, p.521] reads,

'Let $m:=\frac{n(n+1)}{2}$, where $n$ is a positive integer. Then, as $n$ approaches infinity,

$$
\begin{aligned}
\sum_{k=1}^{\infty} \frac{1}{k} \sim & \frac{1}{2} \ln (2 m)+\gamma+\frac{1}{12 m}-\frac{1}{120 m^{2}}+\frac{1}{630 m^{3}}-\frac{1}{1,680 m^{4}}+\frac{1}{2,310 m^{5}} \\
& -\frac{191}{360,360 m^{6}}+\frac{1}{30,030 m^{7}}-\frac{2,833}{1,166,880 m^{8}}+\frac{140,051}{17,459,442 m^{9}}-[\cdots] .
\end{aligned}
$$

For the history and the development of Ramanujan's formula, see [20].

Recently, by changing the logarithmic term in (1.1), DeTemple [15], Negoi [18] and Chen et al. [14] have presented, respectively, faster and faster asymptotic formulas as follows:

$$
\begin{aligned}
& \sum_{k=1}^{n} \frac{1}{k}-\ln \left(n+\frac{1}{2}\right)=\gamma+O\left(n^{-2}\right) \quad(n \rightarrow \infty) ; \\
& \sum_{k=1}^{n} \frac{1}{k}-\ln \left(n+\frac{1}{2}+\frac{1}{24 n}\right)=\gamma+O\left(n^{-3}\right) \quad(n \rightarrow \infty) \\
& \sum_{k=1}^{n} \frac{1}{k}-\ln \left(n+\frac{1}{2}+\frac{1}{24 n}-\frac{1}{48 n^{2}}\right)=\gamma+O\left(n^{-4}\right) \quad(n \rightarrow \infty) .
\end{aligned}
$$

Chen and Mortici [13] provided a faster asymptotic formula than those in (1.2) to (1.4),

$$
\sum_{k=1}^{n} \frac{1}{k}-\ln \left(n+\frac{1}{2}+\frac{1}{24 n}-\frac{1}{48 n^{2}}+\frac{23}{5,760 n^{3}}\right)=\gamma+O\left(n^{-5}\right) \quad(n \rightarrow \infty)
$$

and posed the following natural question.

Open problem For a given positive integer $p$, find the constants $a_{j}(j=0,1,2, \ldots, p)$ such that

$$
\sum_{k=1}^{n} \frac{1}{k}-\ln \left(n+\sum_{j=0}^{p} \frac{a_{j}}{n^{j}}\right)
$$

is the sequence which would converge to $\gamma$ in the fastest way.

Very recently, Yang [21] published the solution of the open problem (1.6) by using logarithmic-type Bell polynomials. 
For all $n \in \mathbb{N}$, let

$$
P_{n}=\sum_{k=1}^{n} \frac{1}{k}-\frac{1}{2} \ln \left(n^{2}+n+\frac{1}{3}\right)
$$

and

$$
Q_{n}=\sum_{k=1}^{n} \frac{1}{k}-\frac{1}{4} \ln \left[\left(n^{2}+n+\frac{1}{3}\right)^{2}-\frac{1}{45}\right]
$$

Chen and Li [12] proved that for all integers $n \geq 1$,

$$
\frac{1}{180(n+1)^{4}}<\gamma-P_{n}<\frac{1}{180 n^{4}}
$$

and

$$
\frac{8}{2,835(n+1)^{6}}<Q_{n}-\gamma<\frac{8}{2,835 n^{6}} .
$$

Now we define the sequences

$$
u_{n}=\sum_{k=1}^{n} \frac{1}{k}-\frac{1}{2} \ln \left(n^{2}+n+\frac{1}{3}\right)-\frac{1}{r\left(n^{2}+n+\frac{1}{3}\right)+s\left(n^{2}+n+\frac{1}{3}\right)^{2}+t}
$$

and

$$
\begin{aligned}
v_{n}= & \sum_{k=1}^{n} \frac{1}{k}-\frac{1}{2} \ln \left(n^{2}+n+\frac{1}{3}\right) \\
& -\left(\frac{a}{\left(n^{2}+n+\frac{1}{3}\right)^{2}}+\frac{b}{\left(n^{2}+n+\frac{1}{3}\right)^{3}}+\frac{c}{\left(n^{2}+n+\frac{1}{3}\right)^{4}}+\frac{d}{\left(n^{2}+n+\frac{1}{3}\right)^{5}}\right),
\end{aligned}
$$

respectively. Our Theorems 1 and 2 are to find the values $r, s, t, a, b, c$ and $d$ which provide the fastest sequences $\left(u_{n}\right)_{n \geq 1}$ and $\left(v_{n}\right)_{n \geq 1}$ approximating the Euler-Mascheroni constant.

Theorem 1 Let $\left(u_{n}\right)_{n \geq 1}$ be defined by (1.9). For

$$
r=-\frac{640}{7}, \quad s=-180, \quad t=\frac{26,770}{441},
$$

we have

$$
\lim _{n \rightarrow \infty} n^{11}\left(u_{n}-u_{n+1}\right)=\frac{457,528}{123,773,265}
$$

and

$$
\lim _{n \rightarrow \infty} n^{10}\left(u_{n}-\gamma\right)=\frac{457,528}{123,773,265} .
$$

The speed of convergence of the sequence $\left(u_{n}\right)_{n \geq 1}$ is $n^{-10}$. 
Theorem 2 Let $\left(v_{n}\right)_{n \geq 1}$ be defined by (1.10). For

$$
a=-\frac{1}{180}, \quad b=\frac{8}{2,835}, \quad c=-\frac{5}{1,512}, \quad d=\frac{592}{93,555},
$$

we have

$$
\lim _{n \rightarrow \infty} n^{13}\left(v_{n}-v_{n+1}\right)=-\frac{796,801}{3,648,645} \text { and } \lim _{n \rightarrow \infty} n^{12}\left(v_{n}-\gamma\right)=-\frac{796,801}{43,783,740} .
$$

The speed of convergence of the sequence $\left(v_{n}\right)_{n \geq 1}$ is $n^{-12}$.

Our Theorems 3 and 4 establish the bounds for $\gamma-P_{n}$ in terms of $n^{2}+n+\frac{1}{3}$.

Theorem 3 Let $P_{n}$ be defined by (1.7). Then

$$
\begin{aligned}
& \frac{1}{\frac{640}{7}\left(n^{2}+n+\frac{1}{3}\right)+180\left(n^{2}+n+\frac{1}{3}\right)^{2}} \\
& <\gamma-P_{n}<\frac{1}{\frac{640}{7}\left(n^{2}+n+\frac{1}{3}\right)+180\left(n^{2}+n+\frac{1}{3}\right)^{2}-\frac{26,770}{441}} .
\end{aligned}
$$

Theorem 4 Let $P_{n}$ be defined by (1.7). Then

$$
\begin{gathered}
\frac{\frac{1}{180}}{\left(n^{2}+n+\frac{1}{3}\right)^{2}}-\frac{\frac{8}{2,835}}{\left(n^{2}+n+\frac{1}{3}\right)^{3}}+\frac{\frac{5}{1,512}}{\left(n^{2}+n+\frac{1}{3}\right)^{4}}-\frac{\frac{592}{93,555}}{\left(n^{2}+n+\frac{1}{3}\right)^{5}} \\
<\gamma-P_{n}<\frac{\frac{1}{180}}{\left(n^{2}+n+\frac{1}{3}\right)^{2}}-\frac{\frac{8}{2,835}}{\left(n^{2}+n+\frac{1}{3}\right)^{3}}+\frac{\frac{5}{1,512}}{\left(n^{2}+n+\frac{1}{3}\right)^{4}} .
\end{gathered}
$$

Remark 1 The inequality (1.14) is sharper than (1.8), while the inequality (1.13) is sharper than (1.14).

\section{Lemmas}

Before we prove the main theorems, let us give some preliminary results.

The constant $\gamma$ is deeply related to the gamma function $\Gamma(z)$ thanks to the Weierstrass formula:

$$
\Gamma(z)=\frac{e^{-\gamma z}}{z} \prod_{k=1}^{\infty}\left\{\left(1+\frac{z}{k}\right)^{-1} e^{z / k}\right\} \quad\left(z \in \mathbb{C} \backslash Z_{0}^{-} ; Z_{0}^{-}:=\{-1,-2,-3, \ldots\}\right) .
$$

The logarithmic derivative of the gamma function

$$
\psi(z)=\frac{\Gamma^{\prime}(z)}{\Gamma(z)} \quad \text { or } \quad \ln \Gamma(z)=\int_{1}^{z} \psi(t) \mathrm{d} t
$$

is known as the psi (or digamma) function. The successive derivatives of the psi function $\psi(z)$

$$
\psi^{(n)}(z):=\frac{\mathrm{d}^{n}}{\mathrm{~d} z^{n}}\{\psi(z)\} \quad(n \in \mathbb{N})
$$

are called the polygamma functions. 
The following recurrence and asymptotic formulas are well known for the psi function:

$$
\psi(z+1)=\psi(z)+\frac{1}{z}
$$

(see [23, p.258]), and

$$
\psi(z) \sim \ln z-\frac{1}{2 z}-\frac{1}{12 z^{2}}+\frac{1}{120 z^{4}}-\frac{1}{252 z^{6}}+\cdots \quad(z \rightarrow \infty \text { in }|\arg z|<\pi)
$$

(see [23, p.259]). From (2.1) and (2.2), we get

$$
\psi(n+1) \sim \ln n+\frac{1}{2 n}-\frac{1}{12 n^{2}}+\frac{1}{120 n^{4}}-\frac{1}{252 n^{6}}+\cdots \quad(n \rightarrow \infty) .
$$

It is also known [23, p.258] that

$$
\psi(n+1)=-\gamma+\sum_{k=1}^{n} \frac{1}{k}
$$

Lemma $1[24,25]$ If $\left(\lambda_{n}\right)_{n \geq 1}$ is convergent to zero and there exists the limit

$$
\lim _{n \rightarrow \infty} n^{k}\left(\lambda_{n}-\lambda_{n+1}\right)=l \in \mathbb{R}
$$

with $k>1$, then there exists the limit

$$
\lim _{n \rightarrow \infty} n^{k-1} \lambda_{n}=\frac{l}{k-1}
$$

Lemma 1 gives a method for measuring the speed of convergence.

Lemma 2 [26, Theorem 9] Let $k \geq 1$ and $n \geq 0$ be integers. Then, for all real numbers $x>0$,

$$
S_{k}(2 n ; x)<(-1)^{k+1} \psi^{(k)}(x)<S_{k}(2 n+1 ; x),
$$

where

$$
S_{k}(p ; x)=\frac{(k-1) !}{x^{k}}+\frac{k !}{2 x^{k+1}}+\sum_{i=1}^{p}\left[B_{2 i} \prod_{j=1}^{k-1}(2 i+j)\right] \frac{1}{x^{2 i+k}}
$$

and $B_{i}(i=0,1,2, \ldots)$ are Bernoulli numbers defined by

$$
\frac{t}{e^{t}-1}=\sum_{i=0}^{\infty} B_{i} \frac{t^{i}}{i !}
$$

It follows from (2.4) that for $x>0$,

$$
\begin{aligned}
\frac{1}{x} & +\frac{1}{2 x^{2}}+\frac{1}{6 x^{3}}-\frac{1}{30 x^{5}}+\frac{1}{42 x^{7}}-\frac{1}{30 x^{9}}+\frac{5}{66 x^{11}}-\frac{691}{2,730 x^{13}} \\
& <\psi^{\prime}(x)<\frac{1}{x}+\frac{1}{2 x^{2}}+\frac{1}{6 x^{3}}-\frac{1}{30 x^{5}}+\frac{1}{42 x^{7}}-\frac{1}{30 x^{9}}+\frac{5}{66 x^{11}}-\frac{691}{2,730 x^{13}}+\frac{7}{6 x^{15}}
\end{aligned}
$$


from which we imply that for $x>0$,

$$
\begin{aligned}
\frac{1}{x} & -\frac{1}{2 x^{2}}+\frac{1}{6 x^{3}}-\frac{1}{30 x^{5}}+\frac{1}{42 x^{7}}-\frac{1}{30 x^{9}}+\frac{5}{66 x^{11}}-\frac{691}{2,730 x^{13}} \\
& <\psi^{\prime}(x+1) \\
& <\frac{1}{x}-\frac{1}{2 x^{2}}+\frac{1}{6 x^{3}}-\frac{1}{30 x^{5}}+\frac{1}{42 x^{7}}-\frac{1}{30 x^{9}}+\frac{5}{66 x^{11}}-\frac{691}{2,730 x^{13}}+\frac{7}{6 x^{15}} .
\end{aligned}
$$

\section{Proofs of Theorems 1-4}

Proof of Theorem 1 By using the Maple software, we write the difference $u_{n}-u_{n+1}$ as a power series in $n^{-1}$ :

$$
\begin{aligned}
u_{n}-u_{n+1}= & \left(-\frac{s+180}{45 s}\right) \frac{1}{n^{5}}+\left(\frac{s+180}{9 s}\right) \frac{1}{n^{6}} \\
& +\left(\frac{2\left(-6,048 s+567 r-32 s^{2}\right)}{189 s^{2}}\right) \frac{1}{n^{7}} \\
& +\left(\frac{2\left(-567 r+2,268 s+11 s^{2}\right)}{27 s^{2}}\right) \frac{1}{n^{8}} \\
& +\left(\frac{2\left(-23 s^{3}+2,430 s r-5,310 s^{2}+108 s t-108 r^{2}\right)}{27 s^{3}}\right) \frac{1}{n^{9}} \\
& +\left(\frac{2\left(-13,770 s r+19,170 s^{2}-1,620 s t+1,620 r^{2}+73 s^{3}\right)}{45 s^{3}}\right) \frac{1}{n^{10}} \\
& +\frac{1}{2,673 s^{4}}\left(-15,443 s^{4}+4,834,566 s^{2} r-4,650,624 s^{3}+1,033,560 s^{2} t\right. \\
& \left.-1,033,560 s r^{2}-53,460 s r t+26,730 r^{3}\right) \frac{1}{n^{11}} \\
& +O\left(\frac{1}{n^{12}}\right) .
\end{aligned}
$$

According to Lemma 1, we have three parameters $r, s$ and $t$ which produce the fastest convergence of the sequence from (3.1)

$$
\left\{\begin{array}{l}
s+180=0 \\
-6,048 s+567 r-32 s^{2}=0, \\
-23 s^{3}+2,430 s r-5,310 s^{2}+108 s t-108 r^{2}=0
\end{array}\right.
$$

namely if

$$
r=-\frac{640}{7}, \quad s=-180, \quad t=\frac{26,770}{441} .
$$

Thus, we have

$$
u_{n}-u_{n+1}=\frac{457,528}{123,773,265 n^{11}}+O\left(\frac{1}{n^{12}}\right) .
$$

By using Lemma 1, we obtain the assertion of Theorem 1. 
Proof of Theorem 2 By using the Maple software, we write the difference $v_{n}-v_{n+1}$ as a power series in $n^{-1}$ :

$$
\begin{aligned}
v_{n}-v_{n+1}= & \left(-\frac{1}{45}-4 a\right) \frac{1}{n^{5}}+\left(\frac{1}{9}+20 a\right) \frac{1}{n^{6}}+\left(-64 a-6 b-\frac{64}{189}\right) \frac{1}{n^{7}} \\
& +\left(\frac{22}{27}+168 a+42 b\right) \frac{1}{n^{8}}+\left(-\frac{1,180}{3} a-8 c-\frac{46}{27}-180 b\right) \frac{1}{n^{9}} \\
& +\left(72 c+\frac{146}{45}+852 a+612 b\right) \frac{1}{n^{10}} \\
& +\left(-\frac{1,160}{3} c-\frac{15,443}{2,673}-\frac{5,426}{3} b-10 d-\frac{46,976}{27} a\right) \frac{1}{n^{11}} \\
& +\left(\frac{2,375}{243}+\frac{14,542}{3} b+\frac{4,840}{3} c+\frac{91,432}{27} a+110 d\right) \frac{1}{n^{12}}+O\left(\frac{1}{n^{13}}\right) .
\end{aligned}
$$

According to Lemma 1, we have four parameters $a, b, c$ and $d$ which produce the fastest convergence of the sequence from (3.2)

$$
\left\{\begin{array}{l}
-\frac{1}{45}-4 a=0, \\
-64 a-6 b-\frac{64}{189}=0, \\
-\frac{1,180}{3} a-8 c-\frac{46}{27}-180 b=0, \\
-\frac{1,160}{3} c-\frac{15,443}{2,673}-\frac{5,426}{3} b-10 d-\frac{46,976}{27} a=0,
\end{array}\right.
$$

namely if

$$
a=-\frac{1}{180}, \quad b=\frac{8}{2,835}, \quad c=-\frac{5}{1,512}, \quad d=\frac{592}{93,555} .
$$

Thus, we have

$$
v_{n}-v_{n+1}=-\frac{796,801}{3,648,645 n^{13}}+O\left(\frac{1}{n^{14}}\right)
$$

By using Lemma 1, we obtain the assertion of Theorem 2.

Proof of Theorem 3 Here we only prove the second inequality in (1.13). The proof of the first inequality in (1.13) is similar. The upper bound of (1.13) is obtained by considering the function $F$ for $x \geq 1$ defined by

$$
F(x)=\frac{1}{2} \ln \left(x^{2}+x+\frac{1}{3}\right)-\psi(x+1)-\frac{1}{\frac{640}{7}\left(n^{2}+n+\frac{1}{3}\right)+180\left(n^{2}+n+\frac{1}{3}\right)^{2}-\frac{26,770}{441}} .
$$

Differentiation and applying the right-hand inequality of (2.5) yield

$$
\begin{aligned}
F^{\prime}(x)= & -\psi^{\prime}(x+1)+\frac{2 x+1}{2\left(x^{2}+x+\frac{1}{3}\right)} \\
& +\frac{55,566\left(126 x^{3}+189 x^{2}+137 x+37\right)}{5\left(7,938 x^{4}+15,876 x^{3}+17,262 x^{2}+9,324 x-451\right)^{2}}
\end{aligned}
$$




$$
\begin{aligned}
> & -\left(\frac{1}{x}-\frac{1}{2 x^{2}}+\frac{1}{6 x^{3}}-\frac{1}{30 x^{5}}+\frac{1}{42 x^{7}}-\frac{1}{30 x^{9}}+\frac{5}{66 x^{11}}-\frac{691}{2,730 x^{13}}+\frac{7}{6 x^{15}}\right) \\
& +\frac{2 x+1}{2\left(x^{2}+x+\frac{1}{3}\right)}+\frac{55,566\left(126 x^{3}+189 x^{2}+137 x+37\right)}{5\left(7,938 x^{4}+15,876 x^{3}+17,262 x^{2}+9,324 x-451\right)^{2}} \\
= & \frac{P(x)}{30,030 x^{13}\left(3 x^{2}+3 x+1\right)^{6}},
\end{aligned}
$$

where

$$
\begin{aligned}
P(x)= & 35,471,898,974,548,627,145+138,773,138,144,376,345,519(x-4) \\
& +241,909,257,272,859,643,240(x-4)^{2} \\
& +253,899,751,881,744,791,655(x-4)^{3} \\
& +181,059,030,163,487,870,836(x-4)^{4} \\
& +93,303,260,620,236,720,571(x-4)^{5} \\
& +35,932,291,146,874,735,228(x-4)^{6}+10,519,794,292,714,982,599(x-4)^{7} \\
& +2,353,926,972,956,528,576(x-4)^{8}+400,626,844,002,342,775(x-4)^{9} \\
& +51,041,813,866,867,916(x-4)^{10}+4,719,218,347,433,667(x-4)^{11} \\
& +299,247,577,164,158(x-4)^{12}+11,646,155,626,560(x-4)^{13} \\
& +209,840,641,920(x-4)^{14}>0 \quad \text { for } x \geq 4 .
\end{aligned}
$$

Therefore, $F^{\prime}(x)>0$ for $x \geq 4$.

For $x=1,2,3,4$, we compute directly:

$$
\begin{aligned}
& F(1)=-0.000018306, \quad F(2)=-2.171 \times 10^{-7}, \\
& F(3)=-1.0 \times 10^{-8}, \quad F(4)=-1.0 \times 10^{-9} .
\end{aligned}
$$

Hence, the sequence $(F(n))_{n \geq 1}$ is strictly increasing. This leads to

$$
F(n)<\lim _{n \rightarrow \infty} F(n)=0
$$

by using the asymptotic formula (2.3). This completes the proof of the second inequality in (1.13).

Proof of Theorem 4 Here we only prove the first inequality in (1.14). The proof of the second inequality in (1.14) is similar. The lower bound of (1.14) is obtained by considering the function $G$ for $x \geq 1$ defined by

$$
\begin{aligned}
G(x)= & \psi(x+1)-\frac{1}{2} \ln \left(x^{2}+x+\frac{1}{3}\right) \\
& +\left(\frac{\frac{1}{180}}{\left(x^{2}+x+\frac{1}{3}\right)^{2}}+\frac{-\frac{8}{2,835}}{\left(x^{2}+x+\frac{1}{3}\right)^{3}}+\frac{\frac{5}{1,512}}{\left(x^{2}+x+\frac{1}{3}\right)^{4}}+\frac{-\frac{592}{93,555}}{\left(x^{2}+x+\frac{1}{3}\right)^{5}}\right) .
\end{aligned}
$$


Differentiation and applying the left-hand inequality of (2.5) yield

$$
\begin{aligned}
G^{\prime}(x)= & \psi^{\prime}(x+1)-\frac{2 x+1}{2\left(x^{2}+x+\frac{1}{3}\right)} \\
& -\frac{3\left(4,158 x^{7}+14,553 x^{6}+19,701 x^{5}+12,870 x^{4}+8,283 x^{3}+6,831 x^{2}-8,276 x-5,194\right)}{770\left(3 x^{2}+3 x+1\right)^{6}} \\
> & \left(\frac{1}{x}-\frac{1}{2 x^{2}}+\frac{1}{6 x^{3}}-\frac{1}{30 x^{5}}+\frac{1}{42 x^{7}}-\frac{1}{30 x^{9}}+\frac{5}{66 x^{11}}-\frac{691}{2,730 x^{13}}\right)-\frac{2 x+1}{2\left(x^{2}+x+\frac{1}{3}\right)} \\
& -\frac{3\left(41,58 x^{7}+14,553 x^{6}+19,701 x^{5}+12,870 x^{4}+8,283 x^{3}+6,831 x^{2}-8,276 x-5,194\right)}{770\left(3 x^{2}+3 x+1\right)^{6}} \\
= & \frac{Q(x)}{30,030 x^{13}\left(3 x^{2}+3 x+1\right)^{6}},
\end{aligned}
$$

where

$$
\begin{aligned}
Q(x)= & 274,317,996,839,484+1,074,684,262,984,527(x-5) \\
& +1,571,352,927,565,772(x-5)^{2}+1,266,557,271,610,345(x-5)^{3} \\
& +652,427,951,634,329(x-5)^{4}+230,639,944,842,034(x-5)^{5} \\
& +57,987,546,990,473(x-5)^{6}+10,515,845,175,406(x-5)^{7} \\
& +1,371,027,303,124(x-5)^{8}+125,702,024,549(x-5)^{9} \\
& +7,709,579,845(x-5)^{10}+284,457,957(x-5)^{11} \\
& +4,780,806(x-5)^{12}>0 \quad \text { for } x \geq 5 .
\end{aligned}
$$

Therefore, $G^{\prime}(x)>0$ for $x \geq 5$.

For $x=1,2,3,4,5$, we compute directly:

$$
\begin{aligned}
& G(1)=-0.000046245 \ldots, \quad G(2)=-1.799 \times 10^{-7}, \quad G(3)=-4 \times 10^{-9}, \\
& G(4)=-1 \times 10^{-9}, \quad G(5)=-1 \times 10^{-10} .
\end{aligned}
$$

Hence, the sequence $(G(n))_{n \geq 1}$ is strictly increasing. This leads to

$$
G(n)<\lim _{n \rightarrow \infty} G(n)=0
$$

by using the asymptotic formula (2.3). This completes the proof of the first inequality in (1.14).

Remark 2 Some calculations in this work were performed by using the Maple software for symbolic calculations.

Remark 3 The work of the first author was supported by a grant of the Romanian National Authority for Scientific Research, CNCS-UEFISCDI, project number PN-II-IDPCE-2011-3-0087. 


\section{Competing interests}

The authors declare that they have no competing interests.

\section{Authors' contributions}

$C M$ proposed the sequence $u_{n}$. CPC proposed the sequence $v_{n}$. CM proposed to solve the problems using Lemma 1 , while CPC used Lemma 2 in evaluations. Both authors made the computations and verified their corectedness. The authors read and approved the final manuscript.

\section{Author details}

${ }^{1}$ Department of Mathematics, Valahia University of Târgovişte, Bd. Unirii 18, Târgovişte, 130082, Romania. ${ }^{2}$ School of Mathematics and Informatics, Henan Polytechnic University, Jiaozuo City, Henan Province 454000, China.

\section{Acknowledgements}

Dedicated to Professor Hari M Srivastava.

Received: 9 December 2012 Accepted: 12 April 2013 Published: 3 May 2013

\section{References}

1. Alzer, H: Inequalities for the gamma and polygamma functions. Abh. Math. Semin. Univ. Hamb. 68, 363-372 (1998)

2. Anderson, GD, Barnard, RW, Richards, KC, Vamanamurthy, MK, Vuorinen, M: Inequalities for zero-balanced hypergeometric functions. Trans. Am. Math. Soc. 347, 1713-1723 (1995)

3. Rippon, PJ: Convergence with pictures. Am. Math. Mon. 93, 476-478 (1986)

4. Tims, SR, Tyrrell, JA: Approximate evaluation of Euler's constant. Math. Gaz. 55, 65-67 (1971)

5. Tóth, L: Problem E3432. Am. Math. Mon. 98, 264 (1991)

6. Tóth, L: Problem E3432 (solution). Am. Math. Mon. 99, 684-685 (1992)

7. Young, RM: Euler's constant. Math. Gaz. 75, 187-190 (1991)

8. Cesàro, E: Sur la serie harmonique. Nouvelles Ann. Math. 4, 295-296 (1885)

9. Chen, C-P: The best bounds in Vernescu's inequalities for the Euler's constant. RGMIA Res. Rep. Coll. 12, Article 11 (2009). Available online at http://ajmaa.org/RGMIA/v12n3.php

10. Chen, C-P: Inequalities and monotonicity properties for some special functions. J. Math. Inequal. 3, 79-91 (2009)

11. Chen, C-P: Inequalities for the Euler-Mascheroni constant. Appl. Math. Lett. 23, 161-164 (2010)

12. Chen, C-P, Li, L: Two accelerated approximations to the Euler-Mascheroni constant. Sci. Magna 6, 102-110 (2010)

13. Chen, C-P, Mortici, C: New sequence converging towards the Euler-Mascheroni constant. Comput. Math. Appl. 64 391-398 (2012)

14. Chen, C-P, Srivastava, HM, Li, L, Manyama, S: Inequalities and monotonicity properties for the psi (or digamma) function and estimates for the Euler-Mascheroni constant. Integral Transforms Spec. Funct. 22, 681-693 (2011)

15. DeTemple, DW: A quicker convergence to Euler's constant. Am. Math. Mon. 100, 468-470 (1993)

16. Mortici, C: On new sequences converging towards the Euler-Mascheroni constant. Comput. Math. Appl. 59 2610-2614 (2010)

17. Mortici, C: Improved convergence towards generalized Euler-Mascheroni constant. Appl. Math. Comput. 215, 3443-3448 (2010)

18. Negoi, T: A faster convergence to the constant of Euler. Gaz. Mat., Ser. A 15, 111-113 (1997) (in Romanian)

19. Vernescu, A: A new accelerated convergence to the constant of Euler. Gaz. Mat., Ser. A 96(17), 273-278 (1999) (in Romanian)

20. Villarino, M: Ramanujan's harmonic number expansion into negative powers of a triangular number. J. Inequal. Pure Appl. Math. 9, Article 89 (2008). Available online at http://www.emis.de/journals/JPAM/images/245_07_JIPAM/245_07.pdf

21. Yang, S: On an open problem of Chen and Mortici concerning the Euler-Mascheroni constant. J. Math. Anal. Appl. 396, 689-693 (2012)

22. Berndt, B: Ramanujan's Notebooks, vol. 5. Springer, New York (1998)

23. Abramowitz, M, Stegun, IA (eds.): Handbook of Mathematical Functions with Formulas, Graphs, and Mathematical Tables. Applied Mathematics Series, vol. 55, 9th printing. National Bureau of Standards, Washington (1972)

24. Mortici, C: New approximations of the gamma function in terms of the digamma function. Appl. Math. Lett. 23, 97-100 (2010)

25. Mortici, C: Product approximations via asymptotic integration. Am. Math. Mon. 117, 434-441 (2010)

26. Alzer, H: On some inequalities for the gamma and psi functions. Math. Comput. 66, 373-389 (1997)

doi:10.1186/1029-242X-2013-222

Cite this article as: Mortici and Chen: On the harmonic number expansion by Ramanujan. Journal of Inequalities and Applications 2013 2013:222. 